\title{
Regulation of Protein Function: Crystal Packing Interfaces and Conformational Dimerization
}

\author{
Peter B. Crowley, Pedro M. Matias, Hualing Mi, Susan Firbank, \\ Mark J. Banfield, and Christopher Dennison
}

Supporting Information

Bridging Ions: Crystallisation of L14F Pc in the presence of $0.2 \mathrm{M} \mathrm{MgCl}_{2}$ resulted in a monomeric arrangement with a $\left[\mathrm{Mg}\left(\mathrm{H}_{2} \mathrm{O}\right)_{6}\right]^{2+}$ complex bound to the protein surface. One of the water molecules from this complex simultaneously hydrogen bonds to the carbonyl oxygen of Pro25 and to a carboxylate oxygen from Asp27 [note that the Asp27 carboxylate does not directly chelate the $\mathrm{Mg}^{2+}$ ion as this would involve the energetically unfavourable process of desolvation (1)]. Despite substantial differences in crystal packing, the hydrogen bonding pattern of Asp27 is the same in all three structures. The $\mathrm{O}^{\delta 2}$ atom is hydrogen-bonded to the amide of His 24 and to a water molecule, whilst the $\mathrm{O}^{\delta 1}$ is hydrogen-bonded to the amino terminus as well as to two water molecules. In the Monomer structure one of these water molecules is replaced by a water molecule coordinated to $\mathrm{Mg}^{2+}$. The $\left[\mathrm{Mg}\left(\mathrm{H}_{2} \mathrm{O}\right)_{6}\right]^{2+}$ complex also acts as a weak bridging ion in the crystal packing interface, via water-mediated hydrogen bonds to the hydroxyl and carbonyl oxygen of Thr22 on a neighbouring monomer.

The role of bridging ions is greater in the Trimer structure where zinc directly facilitates the formation of protein-protein contacts [this is also the case for the WT structure 1baw, $2.8 \AA$ 
resolution (2)]. Each trimer interface contains a $\mathrm{Zn}^{2+}$ ion co-ordinated by the imidazole of His61 and the carboxylates of Asp44 (as a bidentate ligand) and Asp45 (monodentate) from the neighbouring monomer. Two water molecules complete the distorted octahedral co-ordination of the $\mathrm{Zn}^{2+}$ ion. An additional zinc-mediated interface, which was not observed in the WT structure, is present between chains $\mathrm{A}$ and $\mathrm{C}$. This $\mathrm{Zn}^{2+}$ site is co-ordinated by the imidazole of His 24 from two different protein monomers and by the carboxylate of Glu104 (as a bidentate ligand). Again, two water molecules complete the co-ordination site. There is no analogous $\mathrm{Zn}^{2+}$ site involving chain $\mathrm{B}$ and the $\mathrm{N}^{\varepsilon 2}$ of His24 is displaced by $\sim 2 \AA$ from its position in chains $\mathrm{A}$ and $\mathrm{C}$.

Water Molecules: As discussed above for Asp27, the first solvation layer (water molecules that make direct hydrogen bonds or van der Waal's contacts with the protein) is maintained in the different crystal forms $(3,4)$. Besides waters of hydration a number of buried water molecules are observed in the Pc structures. In all of the structures an internal water molecule is hydrogen-bonded to the hydroxyl of Tyr87 and to the carbonyl oxygens of Pro18 and Ala19. This water molecule is conserved in two other cyanobacterial Pc structures $(5,6)$ [but was observed only in chain $\mathrm{C}$ in the WT protein (2)]. Apparently the size and orientation of the side chain at position B8 [Gough and Chothia numbering (7)] determines whether or not a buried water is present. For example in plant Pcs, B8 is usually a buried Phe or Leu whereas in Phormidium Pc the smaller Val21 creates a cavity, which can be occupied by a water molecule. An additional buried water molecule was found in the vicinity of the copper site in the Dimer and in chains A and B of the Trimer structure. This water molecule is hydrogen-bonded to the carbonyl oxygens of Gly12 and Lys35, makes van der Waal's contacts with Gly8, Phe14, Leu36 and His39 (a ligand to the copper) and may play a role in redox modulation of the copper site (8). 

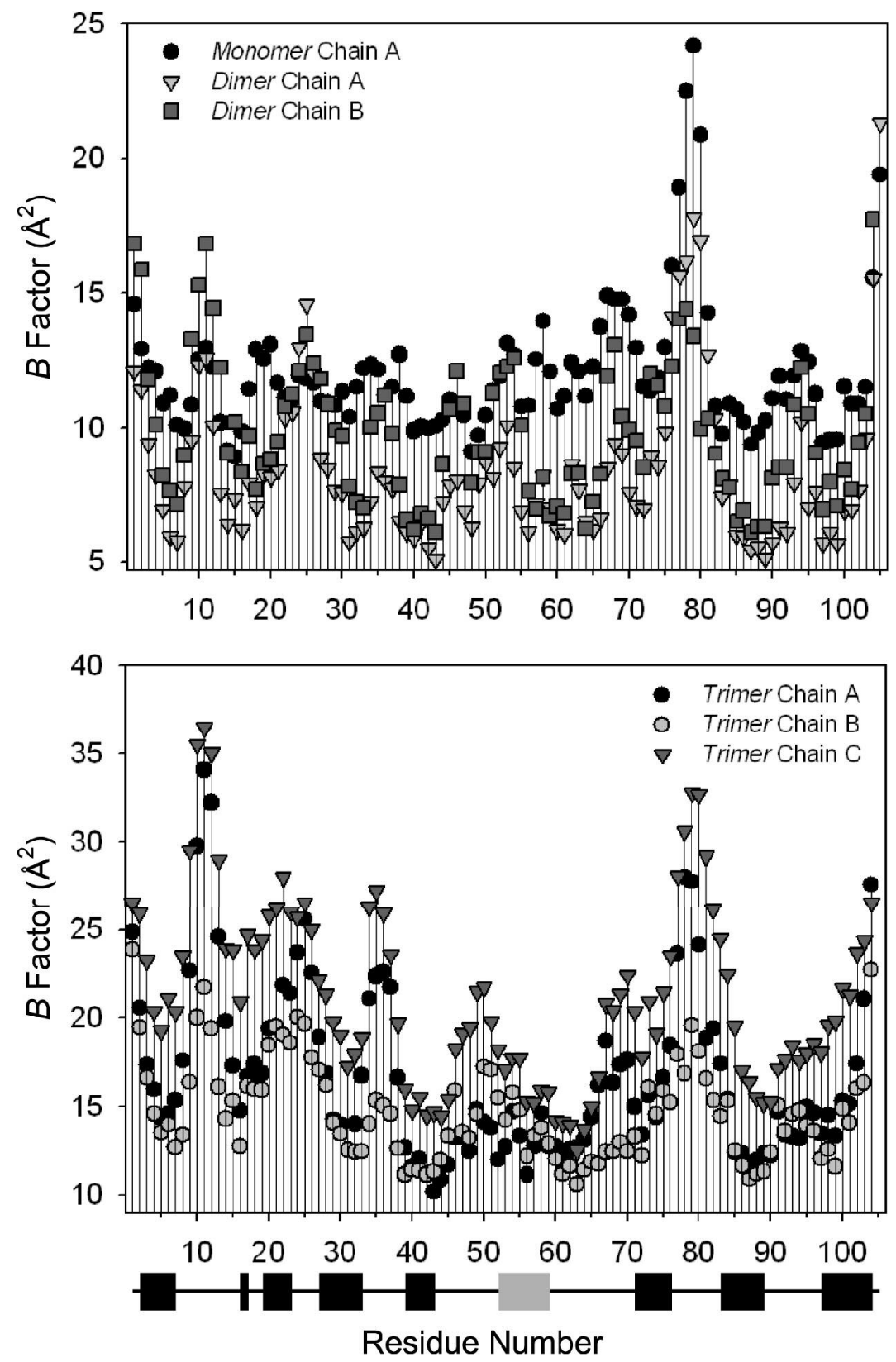

Figure S1. Temperature $(B)$ factors for the $\mathrm{C}^{\alpha}$ atoms in each of the six protein chains in the three crystal forms of L14F Pc. Note the on average higher $B$ values in the Trimer structure. Regions of $\beta$ strand and $\alpha$-helical secondary structure are identified by the black and grey boxes, respectively. As in Figure 1, there is a general pattern of peaks and troughs corresponding to regions of flexible and rigid structure respectively. 


\section{References:}

1. Collins, K. D., and Washabaugh, M. W. (1985) The Hofmeister effect and the behaviour of water at interfaces, Q. Rev. Biophys. 18, 323-422.

2. Bond, C. S., Bendall, D. S., Freeman, H. C., Guss, J. M., Howe, C. J., Wagner, M. J., and Wilce, M. C. J. (1999) The structure of plastocyanin from the cyanobacterium Phormidium laminosum, Acta Cryst. D55, 414-421.

3. Zhang, X. J., and Matthews, B. W. (1994) Conservation of solvent-binding sites in 10 crystal forms of T4 lysozyme, Protein Sci. 3, 1031-1039.

4. Nakasako, M., (2004) Water-protein interactions from high-resolution protein crystallography, Phil. Trans. R. Soc. Lond. B 359, 1191-1206.

5. Romero, A., De la Cerda, B., Varela, P. F., Navarro, J. A., Hervas, M., and De la Rosa, M. A. (1998) The $2.15 \AA$ crystal structure of a triple mutant plastocyanin from the cyanobacterium Synechocystis sp. PCC 6803, J. Mol. Biol. 275, 327-336.

6. Inoue, T., Sugawara, H., Hamanaka, S., Tsukui, H., Suzuki, E., Kohzuma, T., and Kai, Y. (1999) Crystal structure determinations of oxidized and reduced plastocyanin from the cyanobacterium Synechococcus sp. PCC 7942, Biochemistry 38, 6063-6069.

7. Gough, J., and Chothia, C. (2004) The linked conservation of structure and function in a family of high diversity: the monomeric cupredoxins, Structure 12, 917-925.

8. Cascella, M., Magistrato, A., Tavernelli, I., Carloni, P., and Rothlisberger, U. (2006) Role of protein frame and solvent for the redox properties of azurin from Pseudomonas aeruginosa, Proc. Natl. Acad. Sci. USA 103, 19641-19646. 